\title{
Framework for Fusion of Ascending and Descending Pass TanDEM-X Raw DEMs
}

\author{
Rinki Deo, Cristian Rossi, Michael Eineder, Senior Member, IEEE, Thomas Fritz, and Y. S. Rao, Member, IEEE
}

\begin{abstract}
A novel method for calculating optimum incidence angle for the TanDEM-X system using any available digital elevation model (DEM) for the given area is proposed in this study. This method includes the plotting of slopes and aspect of the test area in a statistical way and applying mathematical approach using acquisition geometry in ascending and descending pass TanDEM-X data to optimize the incidence angle for obtaining precise DEM. Furthermore, the TanDEM-X raw DEMs in ascending and descending pass over Mumbai, India are combined using a simple weighted fusion algorithm and the quality of fused DEM thus generated is enhanced. The method adopted for fusion is just an experimental study. The problem of optimum weight selection for fusion has been addressed using height error map and a robust layover shadow mask calculated in "Integrated TanDEM-X Processor" (ITP) during TanDEM-X DEM generation. The height error map is calculated from the interferometric coherence with geometrical considerations and the robust layover and shadow map is calculated using TanDEM-X DEM and the corresponding slant range. Results show a significant reduction in the number of invalid pixels after fusion. In the fused DEM, invalids are only $\mathbf{2 . 1 4 \%}$, while ascending and descending pass DEMs have $5.02 \%$ and $\mathbf{6 . 3 4 \%}$, respectively. Statistical analysis shows a slight improvement in standard deviation of the error in fused DEM by $8 \%$ in urban area and about $5 \%$ for the whole scene. Only slight improvement in accuracy of fused DEM can be attributed to the coarse resolution of the SRTM-X DEM used as reference.
\end{abstract}

Index Terms-Fusion, layover, optimum incidence angle, TanDEM-X digital elevation model (DEM), weightage.

\section{INTRODUCTION}

$\mathbf{T}$ ANDEM-X is the first-ever bistatic satellite mission and capable of providing global digital elevation model (DEM) with an accuracy equaling HRTI-3 standard [1]. The global DEM is being generated using several raw DEMs which are mosaicked and calibrated to achieve this height accuracy [2], [3]. The raw DEMs are generated using single pair acquisition and their quality is affected by many factors such as instrument phase drift [3], baseline errors, interferometric processing errors, and the inherent geometric distortions. The instrument induced errors are removed by intensive instrument calibration and the highly accurate baseline determination helps in removing height biases caused due to baseline errors. For

Manuscript received September 30, 2014; revised April 09, 2015; accepted April 28, 2015.

R. Deo and Y. S. Rao are with the Centre of Studies in Resources Engineering, Indian Institute of Technology Bombay, Mumbai 400076, India (e-mail: rinkideo1@iitb.ac.in; ysrao@iitb.ac.in).

C. Rossi, M. Eineder, and T. Fritz are with the Remote Sensing Technology Institute, German Aerospace Center (DLR), 82234 Wessling, Germany (e-mail: cristian.rossi@dlr.de; michael.eineder@dlr.de; thomas.fritz@dlr.de).

Color versions of one or more of the figures in this paper are available online at http://ieeexplore.iee.org.

Digital Object Identifier 10.1109/JSTARS.2015.2431433 global DEM generation, raw DEMs are further calibrated by applying correction functions and using ICESat data as GCPs [2]. Despite of applying all these calibrations, there still remain inconsistencies in DEM due to inherent geometric distortions, i.e., layover and shadow effect, in complex topographical areas. Layover and shadow are the two very common geometric distortions occurring in SAR image of complex terrain areas. Layover occurs when terrain is steeper than the incidence angle $\left(\theta_{\text {inc }}\right)$. In steep terrains, side-looking viewing property of SAR image causes integration of multiple signals with same range and Doppler frequency within a single resolution cell. Shadow in a SAR image represents zero signal return from the region which is not illuminated by the radar and occurs when terrain back slope is larger than $\left(90^{\circ}-\theta_{\text {inc }}\right)$ [4]. These layover and shadow effects in SAR image cause severe impact on the quality of the reconstructed elevation values [5], [6]. There have been several studies on fusion of DEMs generated using different data sources [7]-[14]. Most of these studies have used the weighted averaging approach for DEM fusion with weight calculated from different methods. Crosetto and Crippa [7] proposed a DEM fusion algorithm to combine optical data with InSAR data. The weights are based on coherence for InSAR and local image correlation for optical images. Papasaika et al. [8] have presented a DEM fusion approach using LiDAR and IKONOS based DEMs. They generated a three-dimensional (3D) error map for each input DEM using their nominal accuracy and geomorphologic characteristics of terrain and then calculated weighted mean based on these error maps. Costantini et al. [9] fused SRTM-X and ERS tandem data with weights depending on individual DEM accuracies. Hoffmann and Walter [10] fused SRTM C- and X-bands with weightage value based on an analysis of the relative differences and the deviations from an absolute reference in a test area. In [11], a method of triangulation of input DEMs is used for DEM fusion with each DEM having a height error map to decide its weightage value. Papasaika et al. [12] presented a generic algorithm to fuse DEMs of different qualities using sparse reconstruction. The mathematical model accounts for prior information if it is available; if no prior information is present, the algorithm calculates some geometrical properties and uses them as weights in the sparse model. Lee et al. [13] has presented a data fusion based on neural network using InSAR, ICESat, and LiDAR DEM. Schultz et al. [14] used self-consistency measures on optical DEMs to identify and remove unreliable elevation estimates (outliers) and then used averaged weighting to fuse the reliable points.

Combination of ascending and descending pass DEMs is one of the promising and widely used techniques for overcoming 
the inherent geometric distortions and fusing InSAR DEMs [11], [15]-[19]. Carrasco et al. [15] presented an algorithm to combine ascending and descending ERS DEMs based on coherence. At each point, the elevation value with higher coherence is chosen as the final DEM value. If both coherences are higher than a set threshold, a weighted average is calculated. A similar weighted average algorithm based on coherence is presented in [16] and [17]. Crosetto [17] also used a second approach of fusion to correct atmospheric errors by using additional DEM of low spatial resolution. A general mosaicking procedure primarily for InSAR DEMs is presented in [18]. The fusion method implements the weighted average approach based on height error maps that represent the quality of each input DEM. In Sansosti et al. [5], coherence was used to mark the unreliable points in both pass ERS tandem DEMs. Using a combination of coherence and baseline values, weights have been assigned to reliable points; finally, the points were averaged according to weights. Jiang et al. [19] used DEMs from TerraSAR-X in ascending and COSMO-SkyMed in descending orbit for a maximum likelihood fusion. The weights were derived from the height of ambiguity and the variance of phase noise. Since the line of sight (LOS) of ascending and descending pass data are symmetrical in nature, the geometrically decorrelated areas in one dataset can be well recovered by the other by combining two, providing that their heading angles are opposite to one another, i.e., the difference in heading angles of ascending and descending passes is close to $180^{\circ}$, so that the terrains are viewed from opposite side. Moreover, for other areas that are geometrically unaffected, the weighted average of two values ensures the stronger contribution from better of the two DEMs and thus reduces the random error of individual DEMs and makes the final DEM more reliable [5]. The simultaneous data acquisition with TanDEM-X mission avoids possible errors from atmospheric disturbance and temporal decorrelations [1] and hence, one of the challenges in TanDEM-X DEM is the detection of unreliable elevation estimates through correct estimation of layover and shadow areas from the data. Since these distortions hardly contain any information about the topography, a quality mask for excluding these areas play an important role in fusing two or more DEMs to generate accurate DEM from SAR images [20]. Another issue in DEM fusion is the selection of optimum weightage value to be given to each dataset while averaging [8], [9]. For an effective fusion of ascending and descending pass DEM in difficult terrain area, it is desired that when the two datasets are combined, the layover areas in one data is well recovered by the other data. In addition to this, each of the two datasets should be acquired with an appropriate incidence angle so as to have minimum layover, as well as shadow effect and most of the slopes in the area are covered. Hence, before applying any intelligent fusion, selection of optimal datasets, capable of giving the best possible DEM after combination, can make the fusion technique more effective and useful. This study focuses on TanDEM$\mathrm{X}$ raw DEMs and has the following structure. In Section II, we give test area and datasets used in this study. A framework is developed in Section III for the selection of optimum TanDEM-X data in ascending and descending pass which can be fused together to obtain more precise DEM. In Section IV,

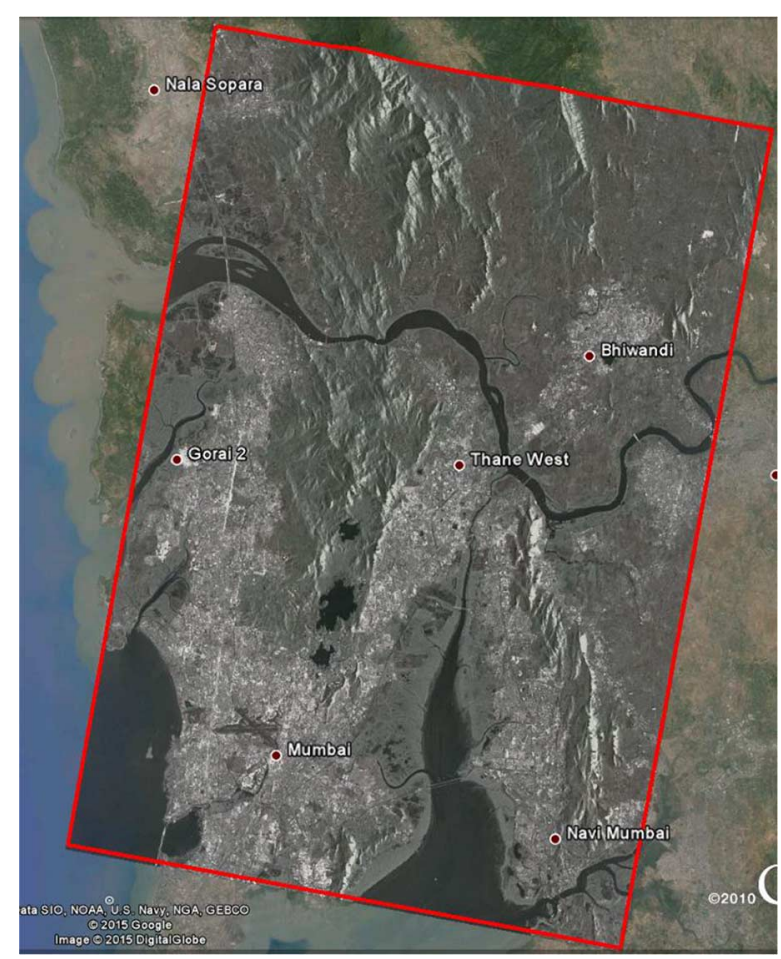

Fig. 1. Amplitude TanDEM-X image of study area of Mumbai overlayed on google earth.

a DEM fusion method using ascending and descending pass TanDEM-X DEMs is proposed to improve the accuracy of final DEM. The accuracy of fused DEM is analyzed in Section V using SRTM-X DEM as reference. Due to nonavailability of any better DEM than the one generated from TanDEM-X data for the test area, we used the SRTM DEM as reference. SRTM X-band DEM which is three times better than that of SRTM C-band in terms of resolution and also better in accuracy [21], [22], was chosen for calibration of TanDEM-X during its processing and also for validation of fused DEM in our study.

\section{Test Site And Data Sources}

The study area is Mumbai situated in Maharashtra state of India. It is in the west coast of India with maximum elevation of $450 \mathrm{~m}$ from the mean sea level. It is one of the most populous urban regions in the world and consists of dense urban settlements, bare land, forest, mangroves, and hilly areas. Within the city, there are three hill ranges and the highest elevation point in the study area lies in one of these hill ranges. The city has also several bays, lakes, and creeks as shown in Fig. 1. TanDEM-X InSAR data in VV-polarization acquired over this area have been used for DEM generation. Table I gives the main parameters of the TanDEM-X data used in this study.

The data were acquired on May 8, 2011 in ascending pass with perpendicular baseline of $161.54 \mathrm{~m}$ and on April 22, 2011 in descending pass with perpendicular baseline of $101.43 \mathrm{~m}$. The corresponding height of ambiguity is 49.21 and $51.47 \mathrm{~m}$, respectively. SRTM X-band DEM is used as reference for accuracy evaluation of the final raw DEM. 
TABLE I

IMPORTANT PARAMETERS OF THE TANDEM-X DATA USED IN THIS STUDY

\begin{tabular}{lll}
\hline \hline Parameters & Asc. pass & Desc. pass \\
\hline Acquisition date & April 22, 2011 & May 8, 2011 \\
Polarization & VV & VV \\
Range spacing (m) & 0.90 & 0.90 \\
Azimuth spacing (m) & 2.03 & 1.96 \\
Heading angle $\left({ }^{\circ}\right)$ & 348.65 & 191.37 \\
Angle of incidence $\left(^{\circ}\right)$ & 46.15 & 33.68 \\
Baseline (perp.) $(\mathrm{m})$ & 161.54 & 101.43 \\
Critical baseline $(\mathrm{m})$ & 4945.6 & 2820.2 \\
Ht. of ambiguity $(\mathrm{m})$ & 49.21 & 51.47 \\
Raw DEM spacing $(\mathrm{m})$ & 6 & 6 \\
\hline \hline
\end{tabular}

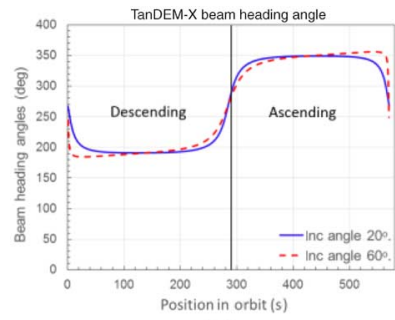

(a)

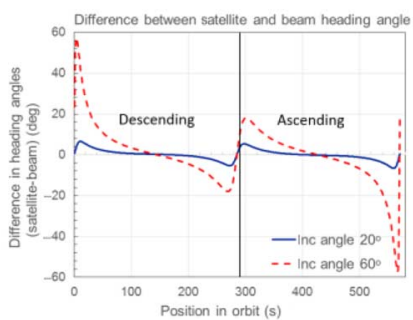

(b)
Fig. 2. (a) TanDEM-X beam heading angles. (b) Difference between satellite and beam heading angles for different latitudes at $20^{\circ}$ and $60^{\circ}$ incidence angles.

\section{FRAMEWORK FOR OPTIMUM TANDEM-X DEM SELECTION}

The data acquisition in either of the passes is governed by viewing geometrical factors such as incidence angle $\left(\theta_{\text {inc }}\right)$ and the heading angle $\left(\theta_{\text {head }}\right) . \theta_{\text {inc }}$ is the angle between the incidence radar beam, i.e., the look direction and the direction perpendicular to the ground surface. The angle between the direction of the satellite velocity and the geographical north, in clockwise direction, is called satellite or system heading angle. For the given satellite system, system heading angle has a fixed value for each of the latitudes. The angle in which beam of the satellite makes with the geographical north is beam heading angle. The beam heading angle depends on latitude as well as on incidence angle and differs from satellite heading angle because of the zero Doppler steering. This difference magnifies toward polar region representing higher beam steering at polar latitudes. Radar mapping in difficult terrain areas are highly affected under varying incidence angle and beam heading angle conditions as these parameters govern the layover and shadow-affected area and the gain in surface area [23]. Fig. 2(a) shows the beam heading angles and Fig. 2(b) shows difference between satellite and beam heading angles for $\theta_{\text {inc }}$ of $20^{\circ}$ and $60^{\circ}$ corresponding to each of the latitudes. Here, latitudes are indicated as satellite position in orbit from 0 to 570 with 285th position corresponding to zero latitude. In Fig. 2(a), we observe that a variation of $40^{\circ}$ in incidence angle corresponds to a change in beam heading angle of $1.8^{\circ}$ for Mumbai area with center latitude $19.2^{\circ}$.

For the radar system to image a particular slope successfully, the facet of the slope must be tilted toward the radar system,

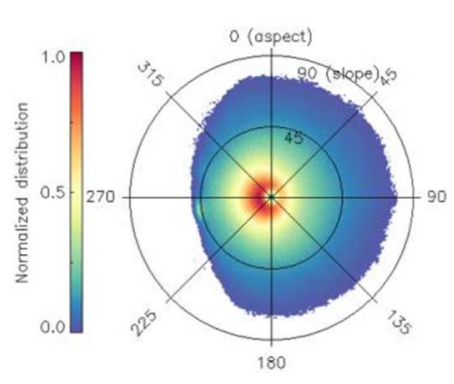

(a)

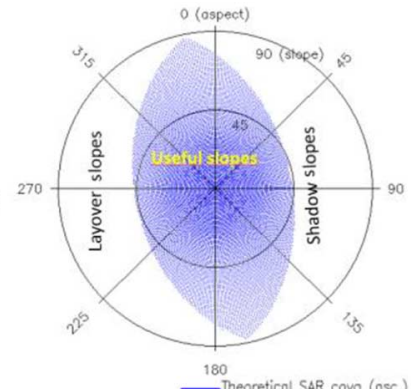

(b)
Fig. 3. (a) Slope and aspect distribution in polar diagram for ascending pass TanDEM-X DEM. (b) Polar plot representing theoretical SAR coverage (in blue) with the ascending pass TanDEM-X data parameters.

such that the angle between look vector and the surface normal vector is less than $90^{\circ}$ given by (1) and the facet should be upright in range direction given by (2) [24]

$$
\begin{aligned}
& \vec{L} \cdot \vec{N}<0 \\
& \vec{M} \cdot \vec{N}>0
\end{aligned}
$$

where $\vec{L}$ is the antenna look vector, $\vec{N}$ is the vector normal to the terrain facet, and $\vec{M}$ is the vector orthogonal to $\vec{L}$ and $\vec{V}$ (antenna velocity vector).

Eineder [24] has theoretically demonstrated the percentage of slope that can be imaged with the given system parameters, i.e., incidence angle and the corresponding heading angle. If we know the slope distribution of a given area along with the system parameter, corresponding percentage of useful slope that can be imaged successfully can also be calculated quantitatively. For this, the slope and aspect values calculated from some available DEM of the area are plotted in polar coordinate as shown in Fig. 3(a) for ascending pass TanDEM-X raw DEM of Mumbai area. The circle in the plot represents all possible combinations of slope and aspect values arranged in polar diagram. Blue-to-red range of color in the plot represents the normalized number of pixels in the data falling under each of these combinations. With any $\theta_{\text {inc }}$ value, corresponding beam heading angle can be calculated and using these parameters, combinations of slope and aspect that can be imaged successfully by the TanDEM-X radar system can also be plotted in polar coordinate as shown in Fig. 3(b). This figure is plotted using the TanDEM-X parameters given in Section II. The blue area within eye-shaped boundary in Fig. 3(b) represents polar diagram of the theoretical successful coverage of TanDEM-X data. This theoretical SAR coverage is nothing but the combination of slope and aspect values which can be imaged successfully with the given TanDEM-X parameters (beam heading angle and incidence angle). It may be noted that this plot represents just a theoretical concept.

When we combine these two polar plots by overlaying TanDEM-X coverage plot, Fig. 3(b) over Fig. 3(a) plot, as shown in Fig. 4(a), we can very easily calculate how many pixels comes within each successful combination of slope and aspect and we further quantify it in terms of percentage of pixels that can be imaged successfully and percentage of pixels that 


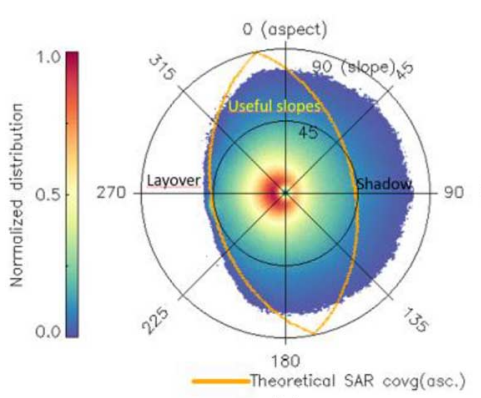

(a)

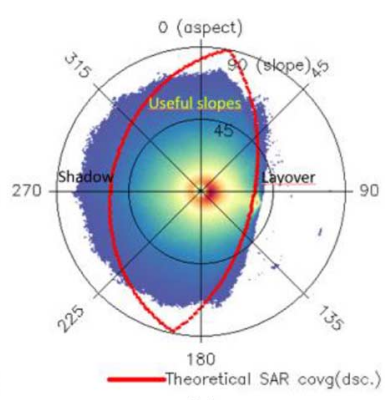

(b)
Fig. 4. Slope/aspect distribution in polar diagram of (a) ascending pass and (b) descending pass TanDEM-X data with theoretical SAR coverage extent (orange and red line) overlaid.

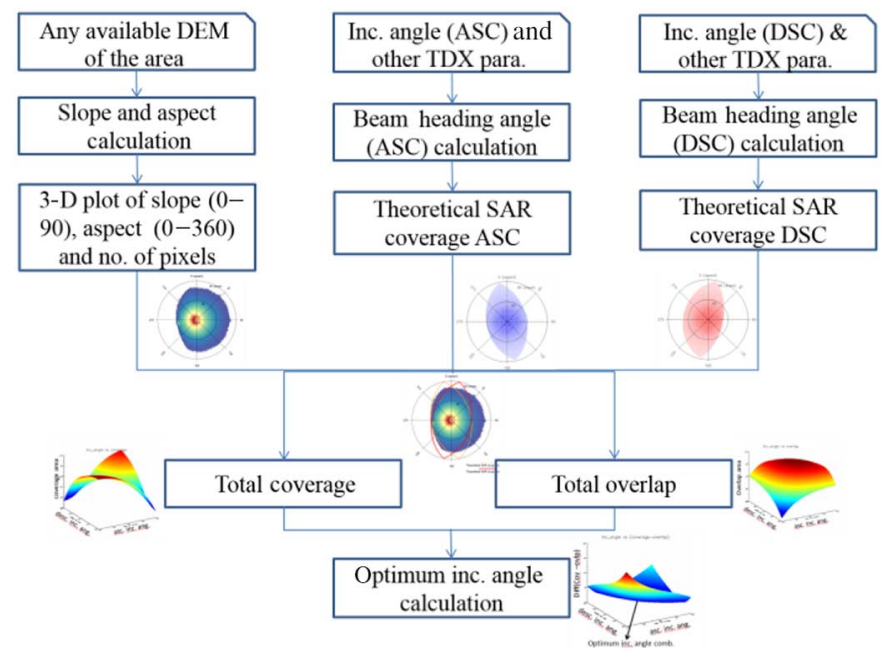

Fig. 5. Flowchart showing the methodology adopted for the calculation of optimum incidence angle for ascending and descending pass TanDEM-X data.

are affected by geometrical distortions. Pixels falling within the eye-shaped (orange color) boundary are the useful slopes that are imaged well in the given data. Pixels lying outside the eyeshaped boundary are the layover and shadow-affected pixels. For the given data, 3.09\% of the pixels are affected by SAR geometric distortions. Similarly, when plotted with descending pass DEM and the satellite parameters with which it is acquired, $3.05 \%$ of the pixels are affected by layover and shadow effect as shown in Fig. 4(b).

We observe from these two plots that the geometrical distortion-affected pixels have opposite aspect value and complement each other to achieve maximum useful slopes when combined. For the given slope and aspect distribution, percentage of useful slopes for different combinations of TanDEM-X observations can be assessed and the optimum combination can be calculated as given in Fig. 5. TanDEM-X data are acquired in the range of $20^{\circ}-60^{\circ}$ incidence angle values in ascending as well as in descending pass. The total area covered and the overlap areas are calculated for all the possible combination of incidence angles in the range of $20^{\circ}-60^{\circ}$ for both the passes.

Fig. 6(a) and (b) shows the two-dimensional (2-D) representation of the total coverage area and total overlap area in percent, respectively, corresponding to all the possible

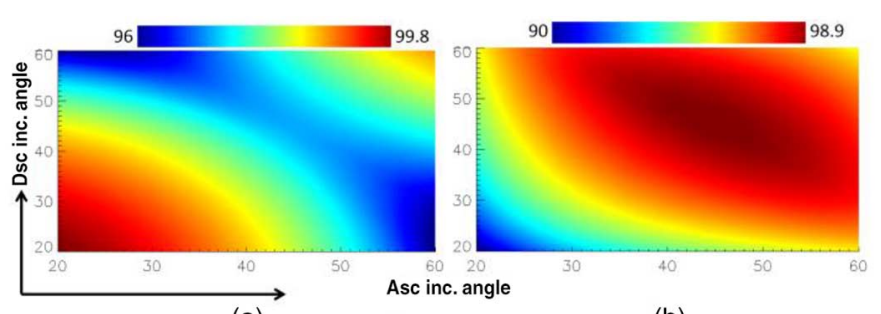

(a)

(b)

Fig. 6. (a) Coverage area and (b) overlap area corresponding to $20^{\circ}-60^{\circ}$ possible combinations of incidence angles in ascending and descending pass using ascending pass TanDEM-X DEM.

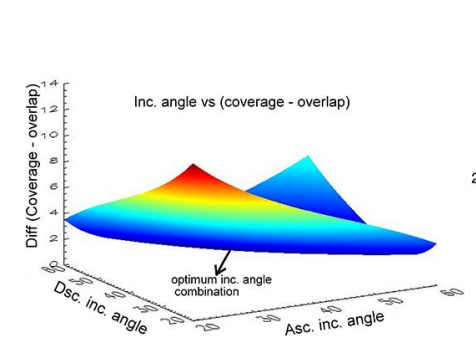

(a)

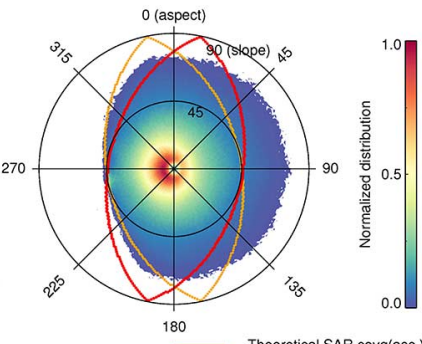

(b) Theoretical SAR covg(asc.)
Theoretical SAR covg(dsc.)

Fig. 7. (a) Combination of incidence angles corresponding to the minimum difference between coverage area and overlap area. (b) Slope/aspect distribution overlaid with theoretical coverage with optimum angles.

combinations of incidence angles in ascending and in descending pass. The color range blue to red represents 96\%-100\% coverage area and $90 \%-98.9 \%$ overlap area. It is observed from this figure that among all the possible combinations, maximum total coverage is achieved for low or high incidence angle combinations, while the overlap area is maximum for angles lying between $40^{\circ}$ and $60^{\circ}$.

In the case of flat areas, combination with maximum coverage is preferred as there are no geometrical decorrelation problems and even individual pass (either asc. or desc.) DEM is reliable enough. However, moderate-to-difficult terrain areas get affected by geometrical distortions and any one of the two pass DEMs cannot be relied much upon due to other inherent source of errors such as baseline errors, instrument phase drift, and interferometric processing errors specially phase unwrapping errors in such areas. Hence, it is preferred to have optimal incidence angle combination which gives maximum coverage as well as maximum overlap area. Maximum coverage helps in ensuring that maximum percentage of slopes of the given complex terrain are imaged successfully by any of the two data passes with minimal layover/shadow effect. Maximum overlap provides us with the choice of keeping the better value of the two available values in the final DEM, thus reducing the random error and making it more reliable and precise compared to individual DEMs. Fig. 7(a) represents the 3-D plot of difference between coverage area and overlap area corresponding to each combination of incidence angles in two passes. The arrow in the figure shows the combination corresponding to the minimum difference and is called as optimum angle combination.

Fig. 7(b) shows the polar plot of theoretical coverage area with optimum incidence angles overlaid on slope/aspect distribution of the test area. For Mumbai area, the optimal 


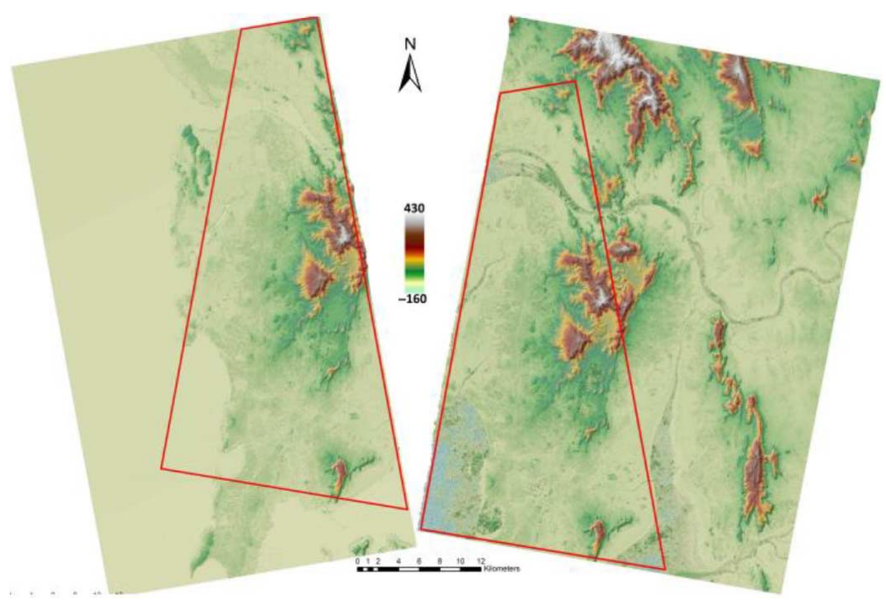

Fig. 8. Ascending and descending pass TanDEM-X raw DEM. Overlaid red boundary is the common area between two datasets.

values calculated are $45^{\circ}$ and $46^{\circ}$ in ascending and descending pass, respectively. Thus, this framework enables us to calculate the percentage of slopes of the given terrain that can be imaged correctly for different observations of the system and hence select the optimum TanDEM-X pair for effective fusion. Slope and aspect distribution can be taken from any available DEM of the area. Higher is the resolution of input DEM, better will be the optimum angle estimation.

\section{Fusion Methodology}

Data acquired with optimal parameters when fused together are expected to give DEM with better accuracy. Due to nonavailability of TanDEM-X data acquired with optimum parameters, the fusion of ascending and descending pass data has been demonstrated using the available data as mentioned in Section II. Here, we note that the angle of incidence for ascending pass data is almost optimum, but for descending pass data, it is much less than the optimum value. Therefore, the result presented from these data in further sections will not be the best possible one as stated above. From the two available datasets, standard raw DEMs with $6 \mathrm{~m}$ spacing were generated independently in the Integrated TanDEM-X Processor (ITP). It is to be noted that in this study, there is no any interference in the standard processing steps for raw DEM generation in ITP. The processor generates DEM for every pixel irrespective of any coherence value. A quality (height error) mask is also provided to recognize bad pixels [3]. Fig. 8 shows the ascending and descending pass TanDEM-X raw DEM over Mumbai area with the common area marked in polygon.

The proposed fusion approach includes generation of a robust layover and shadow mask to be used as one of the inputs. The height error map, which represents the quality of DEM in terms of standard deviation, generated independently during ITP processing for the two pass data along with the DEM and used for optimum weightage calculation. The height error map represents the relative height error $\sigma$, calculated from height of ambiguity $h_{a}$ and the standard deviation of the phase error $\sigma_{\phi}$ corresponding to each pixel of the DEM. The values are assumed to be normally distributed within the image.

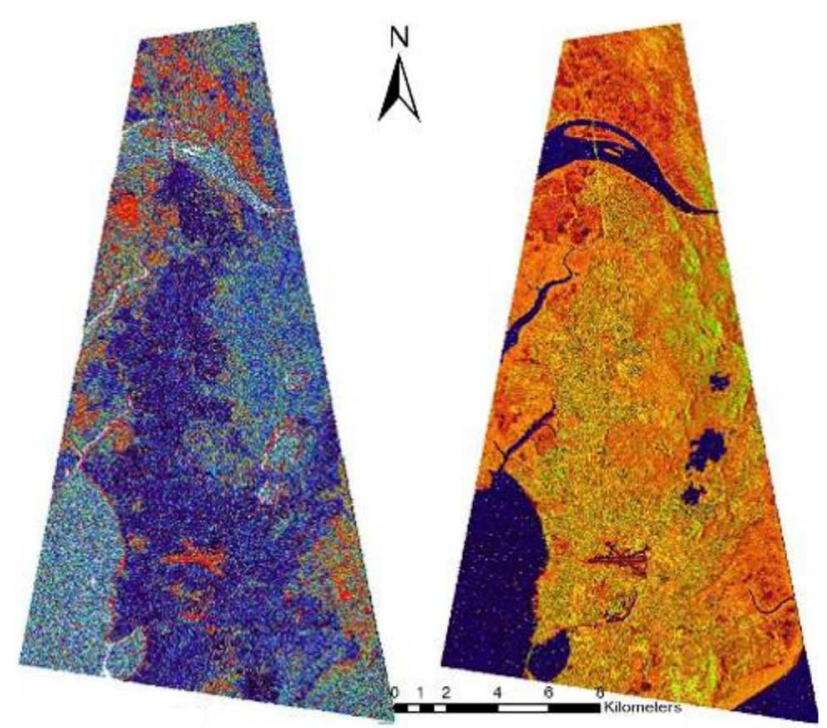

(a)

(b)

Fig. 9. (a) Calculated data statistics layer (window-wise calculation of the ratio of mean and variance of the backscatter value). (b) FCC using coherence (red), backscatter (green), and data statistics (blue) from ascending pass TanDEM-X data.

Height error map for every range and azimuth samples $(\mathrm{x}, \mathrm{y})$ is calculated as

$$
\sigma(x, y)=\sigma_{\varphi}(x, y) \frac{h_{a}}{2 \pi}
$$

where $\sigma_{\phi}(\mathrm{x}, \mathrm{y})$ is the interferometric phase error calculated from the interferometric coherence $(\gamma)$ with geometrical considerations [25]. The layover and shadow mask areas are generated with ITP. In this study, TanDEM-X raw DEM itself is used for robust mask layer generation [26].

\section{A. Fusion of Ascending and Descending Pass InSAR DEMs}

The fusion method proposed in this study is based on height error map and layover and shadow map generated as discussed above. Before proceeding for fusion, the common area between ascending and descending pass data was extracted and then a false color composite (FCC) using coherence, backscatter, and data statistics layer was used to classify and generate water mask area and exclude it from the study area [27]. The data statistics layer represents the window-wise calculation of the ratio of mean and variance of the backscatter value. As the incidence angle for ascending data is close to optimal value, the FCC is generated using ascending pass data. Fig. 9(a) and (b) shows the calculated data statistics layer and generated FCC map, respectively.

Fig. 10(a) and (b) shows the water area masked out ascending and descending pass TanDEM-X DEM for the common area in both the passes on which fusion is applied.

Table II shows the tabular form of the fusion method adopted in this study.

During the process of DEM generation, some spurious height values are obtained in layover and shadow areas. In layover positions, the resulting DEM is modeled with a height ramp [6], whereas shadow areas are seen as random noise in the 


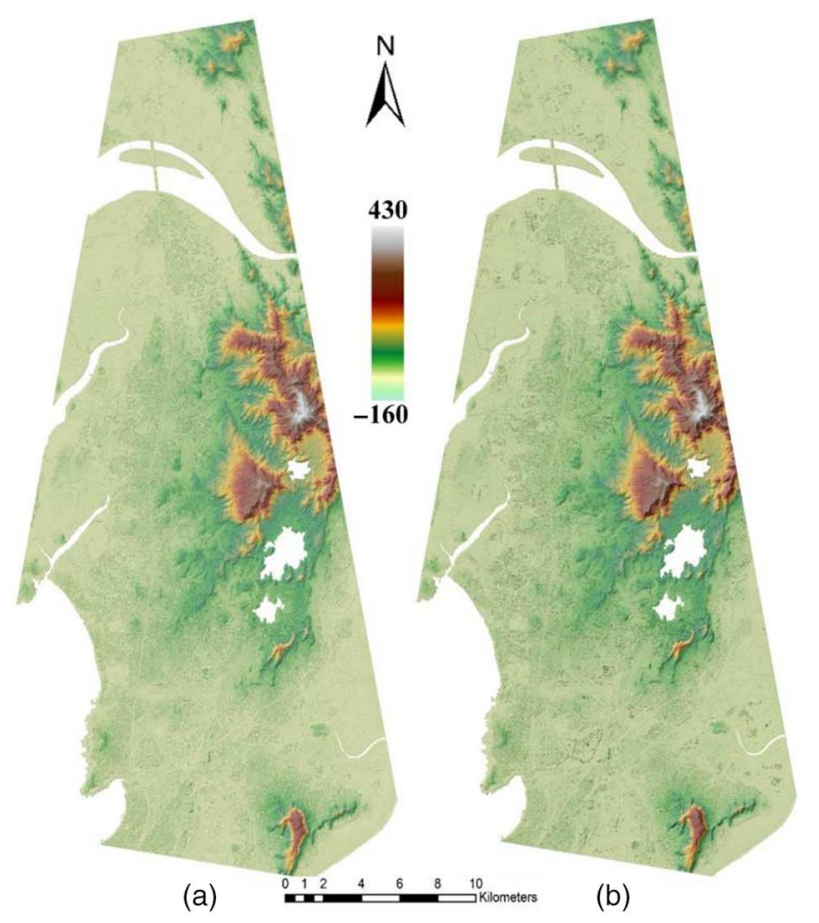

Fig. 10. (a) Ascending and (b) descending pass TanDEM-X raw DEM over Mumbai. Common area in both the passes used for fusion.

TABLE II

Logic For Ascending AND Descending PASS TANDEM-X RAW DEM FUSION

\begin{tabular}{ccccc}
\hline \hline $\begin{array}{c}\text { LS } \\
\text { asc }\end{array}$ & $\begin{array}{c}\text { LS } \\
\text { DSC }\end{array}$ & $\begin{array}{c}\text { HEM } \\
\text { asc }\end{array}$ & $\begin{array}{c}\text { HEM } \\
\text { dsc }\end{array}$ & Fused_DEM value \\
\hline 1 & 1 & - & - & Invalid \\
1 & 0 & - & $>\mathrm{Th}$ & Invalid \\
1 & 0 & - & $<\mathrm{Th}$ & Dsc_DEM value \\
0 & 1 & $>\mathrm{Th}$ & - & Invalid \\
0 & 1 & $<\mathrm{Th}$ & - & Asc_DEM value \\
0 & 0 & $>\mathrm{Th}$ & $>\mathrm{Th}$ & Invalid \\
0 & 0 & $>\mathrm{Th}$ & $<\mathrm{Th}$ & Dsc_DEM value \\
0 & 0 & $<\mathrm{Th}$ & $>\mathrm{Th}$ & Asc_DEM value \\
0 & 0 & $<\mathrm{Th}$ & $<\mathrm{Th}$ & Weighted average \\
\hline \hline
\end{tabular}

DEM due to the lack of information [28]. In the proposed fusion method, various conditions given in Table II are used for obtaining the fused DEM. Pixels affected by layover and shadow in both the DEMs are directly replaced with invalids. Pixels with spurious height values (corresponding HEM value $>$ threshold) in any one of the DEMs are replaced with height values of the other DEM providing that the height error value is less than the threshold value in the other DEM. If this condition is not satisfied, it is replaced with invalids/voids. Pixels free from such distortions in both the DEMs are also checked for replacement with the other DEM value or voids based on their height error values. Finally, pixels with reliable values in both the individual cases are weighted averaged to get the final fused DEM. This simple weighted average approach for fusion of ascending and descending pass InSAR DEM was also used by [5], [15]-[17] using ERS tandem data. However, in these
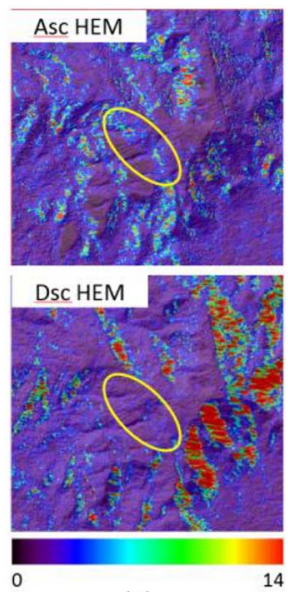

(a)
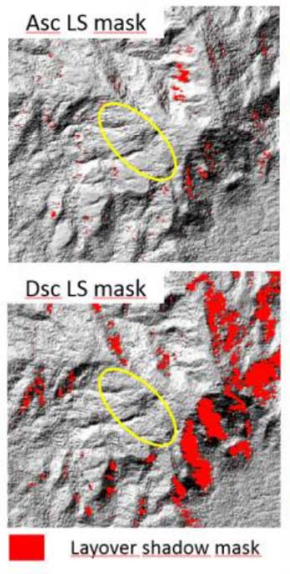

(b)

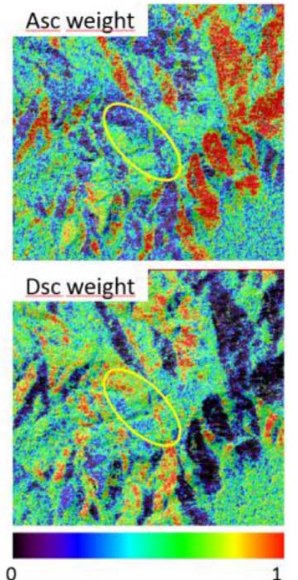

(c)
Fig. 11. (a) HEM for asc. (up) and dsc. (down). (b) Layover-shadow mask for asc. (up) and dsc. (down). (c) Weighatage value map for asc. (up) and dsc. (down) pass data. Area marked in ellipse yellow shows that in geometric distortion free area, weightage value is based on HEM value.

studies, the weightage values are based on coherence with baseline values, only coherence, or variance/covariance matrix of DEM. In our study, height error map used for weightage calculation depends on geometrical factors such as range, incidence angle, and perpendicular baseline which together constitute the height of ambiguity of acquisition and hence represents the result of rigorous error propagation in interferometric phase determination.

According to the distribution of height error values for this area, $95 \%$ of the pixels lie within 2.9 and $3.3 \mathrm{~m}$ of height error in ascending and descending pass DEM, respectively. These values from the height error map are taken as threshold value for deciding the replacements explained above. Weights for both the DEMs are calculated pixel wise from their corresponding height error values as follows:

$$
w_{i}=\frac{1 / \sigma_{i}^{2}}{\sum_{i=1}^{N} 1 / \sigma_{i}^{2}}
$$

where $\sigma_{i}$ is the height error value for ith pixel and $\mathrm{N}=2$ (ascending and descending data) in this case. Finally, the new height value is calculated as

$$
h=\sum_{i=1}^{N} w_{i} h_{i}
$$

where $h_{i}$ is the height value of ith pixel in the two datasets.

Fig. 11 represents the height error map, layover/shadow map, and the weightage map calculated for a small subset of the study area for ascending and descending pass data, respectively. We observe from Fig. 11 that layover/shadow affected areas show lower weightage in both the datasets. Area marked with ellipse is free from geometric distortions in both the datasets and in that case, the weightage value is decided according to the height error value. Since the height error is more in case of ascending data, weightage is given higher value for descending pass data. As a final remark, the algorithm described above is part 


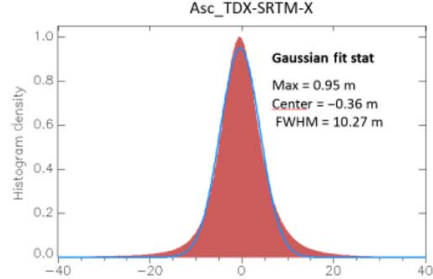

(a)

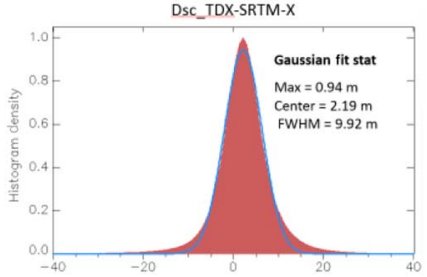

(b)
Fig. 12. Normalized distribution of difference between reference and TanDEM-X. (a) Ascending. (b) Descending DEMs.

of an experimental study and it is not the one operationally employed. Mosaicking process of TanDEM-X data for global DEM generation also uses $\sigma$ value for weightage calculation and the different DEMs are mosaicked by a weighted average of the $\mathrm{N}$ elevation values [2].

\section{RESUlts AND Discussions}

A raw DEM with $6 \mathrm{~m}$ spacing was generated from both ascending as well as descending pass TanDEM-X data acquired in stripmap mode. The height of ambiguity for both the datasets are almost similar $(\sim 50 \mathrm{~m})$. During DEM generation from these datasets, the DEM is calibrated to the SRTM-X band DEM. After extracting the common area and masking out the water, the ascending and descending pass DEM had 5.02\% and 6.34\% of invalids, respectively. These invalids are calculated based on the layover-shadow mask generated and on the threshold value considered using height error map. Higher number of invalids in descending pass DEM can be attributed to its lower incidence angle value and hence, higher number of pixels with slope exceeding this angle within the study area gets affected by layover effect.

Most of the invalids can be seen in the hilly region of the study area which is more prone to geometrical distortions. Fig. 12(a) and (b) shows the normalized distribution of the difference between SRTM-X DEM reference and the generated raw TanDEM-X DEMs in ascending and descending passes, respectively. Sample statistics wise, ascending and descending DEMs show an equivalent root mean square error value of about 7.7 and $7.9 \mathrm{~m}$, respectively. In slope and aspect distribution of the data, $90 \%$ of the slope of the area is within $35.6^{\circ}$ in ascending pass, while using descending pass data, it is $30.9^{\circ}$. This can be attributed to higher number of erroneous pixels in descending pass data due to layover effect in east-facing slope direction. This can be clearly seen as a peak in Fig. 13(a) representing aspect distribution in Cartesian coordinate system of descending pass data, at aspect value of around 100. This peak value is also evident from Fig. 13(b), which represents the polar plot of the slope and aspect value of descending pass TanDEM-X raw DEM.

Fig. 14 shows the ascending DEM, descending DEM, and the fused DEM. It is clearly seen from Fig. 14 that after combining the complementary viewing geometry, the percentage of invalids decreased significantly to $2.14 \%$ compared to the two input DEMs with $5.02 \%$ in ascending and $6.34 \%$ in descending pass. Keeping these $2.14 \%$ invalids common in all the three cases, i.e., in ascending, descending, and fused DEM,

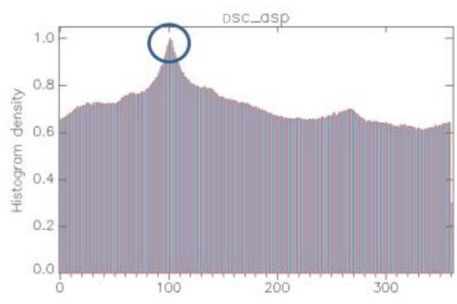

(a)

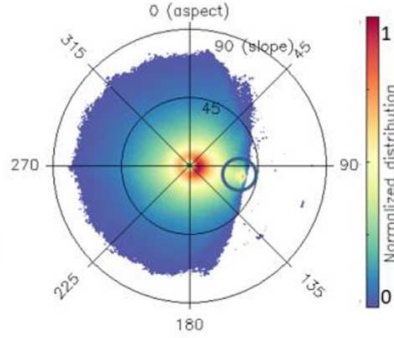

(b)
Fig. 13. Descending pass TanDEM-X DEM. (a) Aspect distribution. (b) Polar diagram of slope and aspect distribution. Circle shows the aspect at which layover effect occurs.

the vertical accuracy has been assessed using SRTM-X band DEM as reference. SRTM-X band DEM with $30 \mathrm{~m}$ resolution was first oversampled to $6 \mathrm{~m}$, equivalent to generated TanDEM$\mathrm{X}$ raw DEM spacing and then the difference between each of these DEMs and the reference DEM was used for error analysis. After DEM fusion, the RMSE decreased to $7.0 \mathrm{~m}$ from $7.3 \mathrm{~m}$ in ascending and $7.5 \mathrm{~m}$ in descending DEM. $90 \%$ absolute error value reduced by $4 \%$ and $90 \%$ relative error value dropped by $6 \%$. Seeing the Gaussian statistics of error distribution, full-width at half maximum (FWHM) shows an improvement by $6 \%$. The height difference between the reference SRTM-X DEM and three DEMs, i.e., ascending, descending, and fused, are quantified as cumulative percentages as done by [19] at six different intervals in Table III.

It is observed from the table that in all the six intervals, ascending pass DEM is better than descending DEM and there is further improvement with fused DEM. The interval $\leq 5.0 \mathrm{~m}$ shows maximum improvement of fused DEM over ascending pass DEM signifying that fused DEM has considerable amount of improvement in pixels with height error more than $\pm 5.0 \mathrm{~m}$. To have a further insight into the accuracy improvement, the study area was classified into urban and nonurban classes using the layer stack of coherence, backscatter, difference backscatter, and data statistics layer discussed in the previous section. The difference backscatter is the difference of backscatter value between master and slave acquisition. Fig. 15 shows the classified map of the study area in urban and nonurban class. It is to be noted that the urban class also includes the layover-affected pixels in hilly area and for this class, the fused DEM shows $8 \%$ drop in the standard deviation value and $8.3 \%$ drop in $90 \%$ absolute error value. The FWHM of the error distribution, which is 11.9 and $11.3 \mathrm{~m}$ in ascending and descending passes, respectively, improves to $10.9 \mathrm{~m}$ in fused DEM.

\section{CONCLUSION}

In this study, a novel method is proposed for calculating optimum incidence angle for the TanDEM-X system using any available DEM. Furthermore, a simple weighted fusion of ascending and descending pass TanDEM-X raw DEMs using height error map and layover/shadow mask is proposed. The most challenging problem of optimum weight selection for fusion has been deciphered using height error value and a robust layover shadow mask generated. The fused DEM 

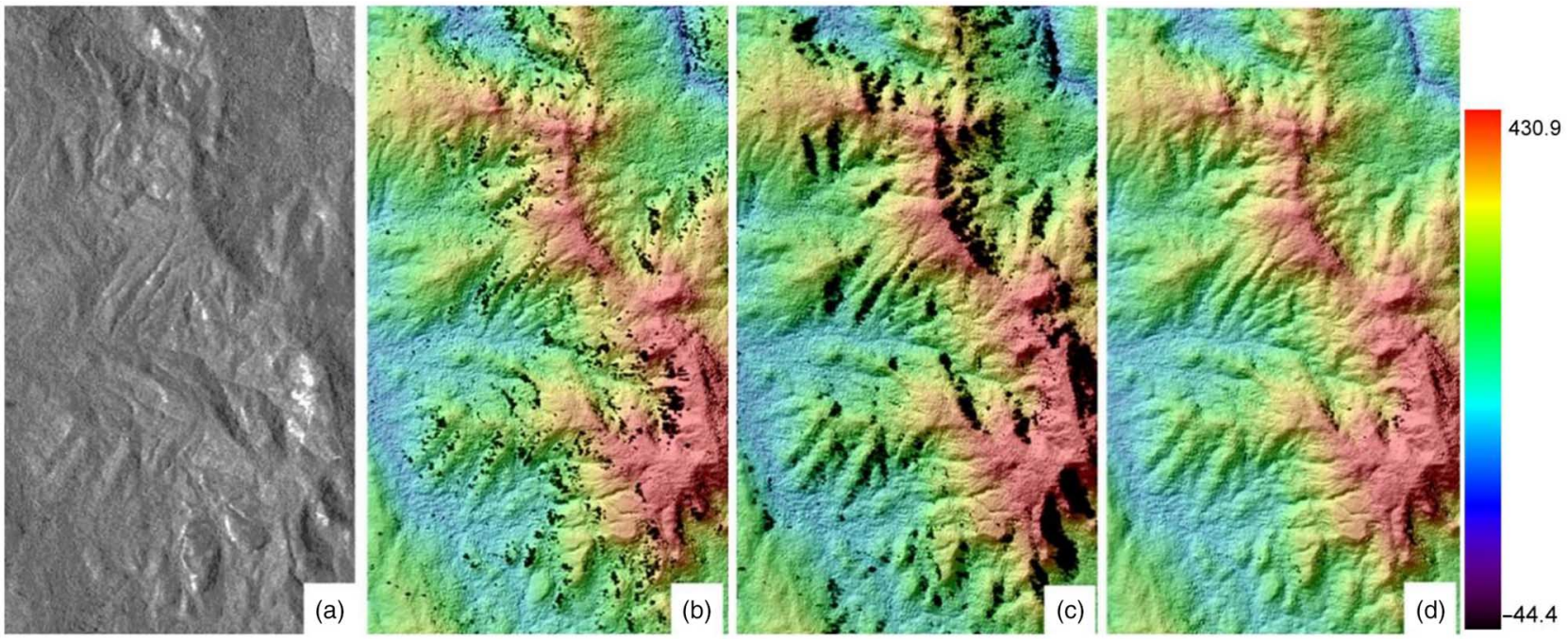

Fig. 14. (a) Amplitude image. (b) Ascending. (c) Descending. (d) Fused DEM. Invalid pixels are shown in black color.

TABLE III

Difference of Height Values in Terms of Cumulative Percentages

\begin{tabular}{lrrrrrr}
\hline \hline & \multicolumn{7}{c}{ Intervals (m) } \\
\hline & $\leq 1.0$ & $\leq 3.0$ & $\leq 5.0$ & $\leq 10.0$ & $\leq 15.0$ & $\leq 20.0$ \\
\cline { 2 - 7 } Ascending DEM & 17.1 & 46.9 & 67.5 & 88.9 & 94.3 & 96.1 \\
Descending DEM & 15.02 & 42.7 & 63.8 & 87.7 & 94.1 & 96.0 \\
Fused DEM & 17.6 & 48.3 & 70.0 & 89.7 & 94.8 & 96.4 \\
\hline \hline
\end{tabular}

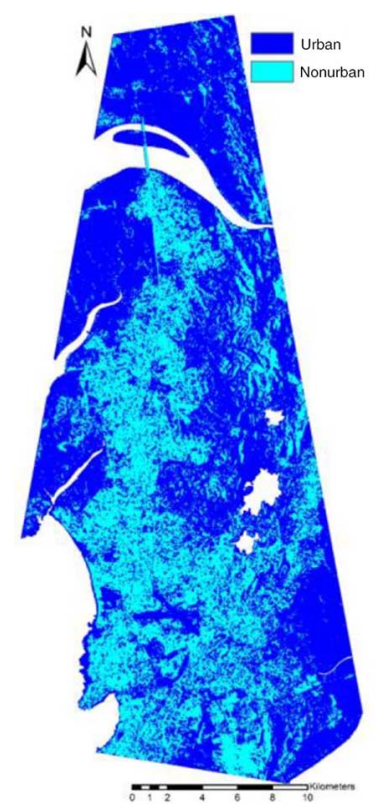

Fig. 15. Study area classified into urban and nonurban class for accuracy assessment.

shows a reduction in the percentage of invalids to $2.14 \%$ compared to $5.02 \%$ and $6.34 \%$ in ascending and descending pass DEMs. Reduction in void pixels in the fused DEM can be seen as major advantage of the fusion method used. However, the disadvantage with the method is that the quality of final output DEM very much depends on the height accuracy map which does not always represent the true quality of the input DEMs. Coherence value just represents the degree of correlation between master and slave image used for generating raw DEM. It does not take into account of other major source of errors such as phase unwrapping errors and elevation offsets in the InSAR processing chain. The accuracy analysis of the final fused DEM has been carried out using SRTM-X DEM. Ascending and descending pass DEM shows comparable accuracy with standard deviation 7.3 and $7.1 \mathrm{~m}$, respectively. Statistical analysis of the accuracy of fused DEM reveals a substantial improvement in standard deviation of the error by $8 \%$ in urban area and about $5 \%$ for the whole scene.

\section{ACKNOWLEDGMENT}

TanDEM-X datasets are obtained from DLR, Germany under AO proposal id XTI_VEGE0635. The authors would like to thank the colleagues M. Fischer and F. Adam for discussions on paper topics. R. Deo thanks DAAD, Germany, for providing fellowship to carry out this work at DLR, Wessling.

\section{REFERENCES}

[1] G. Krieger et al., "TanDEM-X: A satellite formation for high-resolution SAR interferometry," IEEE Trans. Geosci. Remote Sens., vol. 45, no. 11, pp. 3317-3341, Nov. 2007.

[2] A. Gruber, B. Wessel, M. Huber, and A. Roth, "Operational TanDEM$\mathrm{X}$ DEM calibration and first validation results," ISPRS J. Photogramm. Remote Sens., vol. 73, pp. 39-49, 2012.

[3] C. Rossi, F. Gonzalez Rodriguez, T. Fritz, N. Yague Martinez, and M. Eineder, "TanDEM-X calibrated raw DEM generation," ISPRS J. Photogramm. Remote Sens., vol. 73, pp. 12-20, 2012.

[4] W. G. Kropatsch and D. Strobl, "The generation of SAR layover and shadow maps from digital elevation models," IEEE Trans. Geosci. Remote Sens., vol. 28, no. 1, pp. 98-107, Jan. 1990.

[5] E. Sansosti et al., "Digital elevation model generation using ascending and descending ERS-1/ERS-2 tandem data," Int. J. Remote Sens., vol. 20, no. 8, pp. 1527-1547, 1999.

[6] C. Rossi and M. Eineder, "High-resolution InSAR building layovers detection and exploitation," IEEE Trans. Geosci. Remote Sens., to be published.

[7] M. Crosetto and B. Crippa, "Optical and radar data fusion for DEM generation,” IAPRS, vol. 32, no. 4, pp. 128-134, 1998. 
[8] H. Papasaika, D. Poli, and E. Baltsavias, "A framework for the fusion of digital elevation models," in Proc. Int. Arch. Photogramm. Remote Sens. Spat. Inf. Sci., Beijing, China, 2008, vol. 37, part B2, pp. 811-818.

[9] M. Costantini, F. Malvarosa, F. Minati, and E. Zappitelli, "A data fusion algorithm for DEM mosaicking: Building a global DEM with SRTM-X and ERS data," in Proc. Int. Geosci. Remote Sens. Symp. (IGARSS'06), Denver, CO, USA, Jul./Aug. 2006, pp. 3844-3847.

[10] J. Hoffmann and D. Walter, "How complementary are SRTM-X and -C band digital elevation models?" Photogramm. Eng. Remote Sens., vol. 72, no. 3, pp. 261-268, 2006.

[11] D. Hoja, P. Reinartz, and M. Schroeder, "Comparison of DEM generation and combination methods using high resolution optical stereo imagery and interferometric SAR data," in Proc. Int. Soc. Photogramm. Remote Sens. (ISPRS) Comm. I Symp., 2006, vol. 36, pp. 89-94.

[12] H. Papasaika, E. Kokiopoulou, E. Baltsavias, K. Schindler, and D. Kressner, "Fusion of digital elevation models using sparse representations," in Proc. Photogramm. Image Anal., Munich, Germany, 2011, pp. $171-184$

[13] S. H. Lee, S. Baek, and C. K. Shum, "Multi-temporal, multi-resolution data fusion for Antarctica DEM determination using InSAR and Altimetry," in Proc. Int. Geosci. Remote Sens. Symp. (IGARSS'05), 2005, vol. 4, pp. 2827-2829.

[14] H. Schultz, E. M. Riseman, F. R. Stolle, and D. M. Woo, "Error detection and DEM fusion using self-consistency," in Proc. Int. Conf. Comput. Vis., 1999, pp. 1174-1181.

[15] D. Carrasco et al., "Ascending-descending orbit combination SAR interferometry assessment," in Proc. 3rd ERS Symp., Florence, Italy, Mar. 1997, pp. 1789-1794.

[16] M. Crosetto and B. Crippa, "Quality assessment of interferometric DEM," Int. Arch. Photogramm. Remote Sens., vol. 33, part B1, pp. 46-53, 2000.

[17] M. Crosetto, "Calibration and validation of SAR interferometry for DEM generation," ISPRS J. Photogramm. Remote Sens., vol. 57, pp. 213-227, 2002.

[18] W. Knöpfle, G. Strunz, and A. Roth, "Mosaicking of digital elevation models derived by SAR interferometry," Int. Arch. Photogramm. Remote Sens., vol. 32, part 4, pp. 306-313, 1998.

[19] H. Jiang, L. Zhang, Y. Wang, and M. Liao, "Fusion of highresolution DEMs derived from COSMO-SkyMed and TerraSAR-X InSAR datasets," J. Geod., vol. 88, no. 6, pp. 587-599, 2014.

[20] D. Pairman and S. McNeill, "Efficient calculation in the map domain of SAR layover and shadow masks," in Proc. Int. Geosci. Remote Sens. Symp. (IGARSS'97), Aug. 1997, vol. 4, pp. 2057-2059.

[21] U. Marschalk, A. Roth, M. Eineder, and S. Suchandt, "Comparison of DEMs derived from SRTM/X-and C-Band," in Proc. Int. Geosci. Remote Sens. Symp. (IGARSS'04), Sep. 2004, vol. 7, pp. 4531-4534.

[22] T. G. Farr et al., "The shuttle radar topography mission," Rev. Geophys., vol. 45, pp. 1-33, 2007.

[23] M. Eineder and J. Holzner, "Interferometric DEMs in alpine terrainlimits and options for ERS and SRTM," in Proc. Int. Geosci. Remote Sens. Symp. (IGARSS'00), Honolulu Hawaii, Jul. 2000, vol. 7, pp. 3210-3212.

[24] M. Eineder, "Problems and solutions for InSAR digital elevation model generation of mountainous terrain," in Proc. ESA FRINGE Workshop, 2003.

[25] C. Rossi, M. Eineder, T. Fritz, and H. Breit, "TanDEM-X mission: Raw DEM generation," in Proc. 8th Eur. Conf. Synth. Aperture Radar (EUSAR), Jun. 2010, pp. 1-4.

[26] R. Deo et al., "Fusion of ascending and descending pass raw TanDEM-X DEM," in Proc. Int. Geosci. Remote Sens. Symp. (IGARSS'14), Qubec, Canada, Jul. 2014, pp. 21-24.

[27] C. Rossi and E. Erten, "Paddy rice monitoring using TanDEM-X," IEEE Trans. Geosci. Remote Sens., vol. 53, no. 2, pp. 900-910, Feb. 2015.

[28] C. Rossi and S. Gernhardt, "Urban DEM generation, analysis and enhancements using TanDEM-X," ISPRS J. Photogramm. Remote Sens., vol. 85, pp. 120-131, 2013.

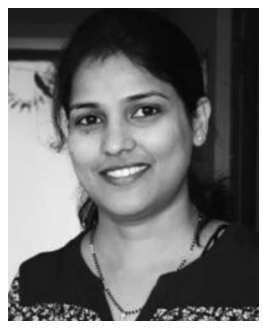

Rinki Deo received the M.Sc. degree in physics from Patna University, Patna, India, in 2006, and the Postgraduate Diploma degree in geoinformatics from IIRS-Dehradun, Dehradun, India, and ITC Netherlands, Enschede, The Netherlands, in 2008. She is currently pursuing the Ph.D. degree in resources engineering at the Indian Institute of Technology Bombay, Mumbai, India.

Her research interests include synthetic aperture radar (SAR), SAR interferometry for DEM generation and its evaluation, polarimetric SAR data classification, and PolInSAR.

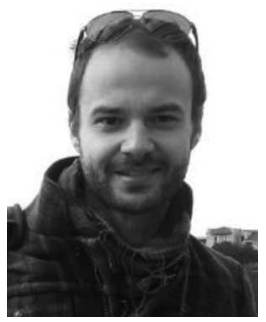

Cristian Rossi received the Laurea Magistrale (M.Sc.) degree in telecommunication engineering from the Politecnico di Milano, Milan, Italy, in 2006.

From 2006 to 2008, he was Project Engineer with Aresys, Milano, Italy. Since 2008, he has been with the SAR Signal Processing Department, Earth Observation Center, German Aerospace Center (DLR), Oberpfaffenhofen, Germany, where he works on the development of the integrated TanDEM-X processor and on novel interferometry algorithms for SAR missions. His research interests include urban remote sensing, multisource data fusion, digital elevation models, and environmental parameter estimation.

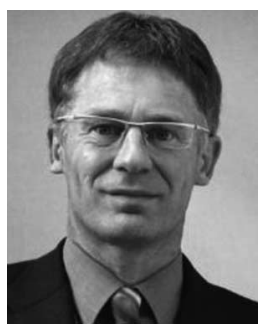

Michael Eineder (SM'01) received the Diploma degree in electrical engineering and telecommunication science from the Technische Universität München, Munich, Germany, and the Dr. rer. nat. degree from the University of Innsbruck, Innsbruck, Austria.

Since 1990, he has been with the German Aerospace Center (DLR), Wessling, Germany, where he gained broad experience in synthetic aperture radar (SAR) algorithm development. He is currently the Head of the SAR Signal Processing Department, DLR. His responsibilities encompass the development of SAR and interferometric SAR processing systems for future radar missions such as TanDEM-X. Furthermore, he is currently a Part-Time Lecturer with the Remote Sensing Department, Technische Universität München. He has worked on a variety of international missions, including SIR-C/X-Band SAR and Shuttle Radar Topography Mission/X-band SAR in cooperation with NASA, European Remote Sensing Satellite-1 (ESA), TerraSAR-X, and TanDEM-X (Germany).

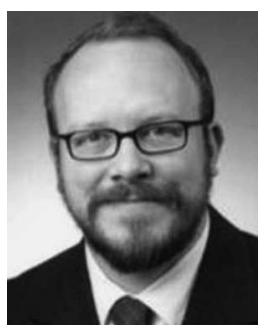

Thomas Fritz received the Diploma degree in physics from the University of Münster, Münster, Germany, in 1996, and the Dr. rer. nat. degree from the University of Bonn, Bonn, Germany, in 2000.

$\mathrm{He}$ was a Postdoctoral Fellow with the Radioastronomical Institute, University of Bonn. Since 2003, he has been with the Remote Sensing Technology Institute, German Aerospace Center (DLR), Wessling, Germany, where he participated in the development of the TerraSAR-X multimode synthetic aperture radar (SAR) processor. In particular, he was responsible for TerraSAR-X SAR product specification, characterization, and verification. He is currently responsible for the development of the interferometric processing chain and the system engineering of the Integrated TanDEM-X processor.

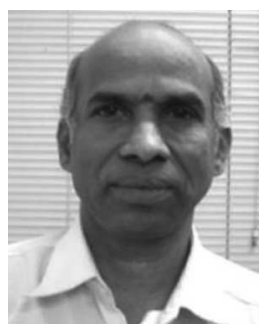

Y. S. Rao (M'06) received the M.Sc. degree in physics from Andhra University, Visakhapatnam, India, in 1982, and the Ph.D. degree in passive microwave remote sensing of soil moisture from the Indian Institute of Technology (IIT) Bombay, Mumbai, India, in 1992.

$\mathrm{He}$ joined the Centre of Studies Resources Engineering, IIT Bombay, in 1985, as a Senior Research Assistant and then became a Research Scientist in 1999. During 2005-2009, he was a Senior Research Scientist and then Associate Professor from 2009 to 2014. He is currently continuing as a Professor. He worked in both passive and active microwave remote sensing for several applications viz. soil moisture, flood mapping, and land use/land cover. He has participated in several spaceborne campaigns for collecting synchronous ground-truth data and has experience in handling various datasets for several applications. His research interests include application of polarimetry for geophysical parameter retrieval and SAR interferometry for DEM and displacement map generation. 